\title{
Efectos y mediación de un programa de habilidades blandas a través del desarrollo de la cognición corporizada en estudiantes universitarios
}

\section{Effects and mediation of a soft skills program through the development of cognition incorporated in university students}

\section{RESUMEN}

Las habilidades blandas han recibido una gran atención con respecto a diversos métodos de evaluación. Sin embargo, aún hay pocos estudios sobre métodos estadísticamente significativos para poder entrenarlas. La cognición corporizada permite plantear una teoría de cómo estas se identificarían desde la conciencia de habilidades encarnadas que han de desarrollarse y mantenerlas en un contexto histórico socio-cultural para llevarlas a un nivel superior en etapas mediadas por la interocepción. Esta teoría planteada por el autor del presente informe se ha puesto a prueba en un programa que ha sido aplicado a trece alumnos universitarios y cuyos resultados, contrastados con un grupo control, han sido significativos con respecto a niveles de nerviosismo $(t=-3,379$ Sig. $=, 003)$ Atención plena $(t$ $=4,762$ Sig. $=, 000)$ Niveles de cognición $(t=5,560$ Sig. $=, 000)$ Afrontamiento del estrés académico $(t=$ $2,802 \mathrm{Sig} .=, 014)$ y reacciones al estrés académico $(t=-4,614$ Sig. $=, 000)$. Al buscar los efectos a las reacciones al estrés académico se encontró que los niveles de cognición, variable del programa, no solo las predice mejor ( $R 2$ Lineal $=0,669$ ) sino que actúa como variable mediadora del afrontamiento del estrés académico y las reacciones al estrés académico (BootLLCl=-.7933 BootULCl=-.2247 y efecto indirecto de -.4439). Comprobando la efectividad del programa y la posibilidad de desarrollar esta teoría.

Palabras Claves: Programa de entrenamiento de habilidades, habilidades blandas, cognición corporizada, interocepción

\begin{abstract}
Soft skills have received a great Attention with respect to various methods of evaluation. However, there are still few studies about statistically significant methods for to be able to train them. The embodied cognition allows pose a theory of how these would be identified from the awareness of embodied abilities that have to be developed and maintained in a context socio-cultural history to bring them to a level superior in stages mediated by interoception. This theory put forward by the author of the present report has been put to the test in a program that has been applied to thirteen university students and whose results, contrasted with a control group, have been significant with respect to levels of nervousness $(t=-3,379$ Sig. $=, 003)$ Mindfulness $(t=4,762$ Sig. $=, 000)$ Levels of cognition $(t=5,560$ Sig. $=, 000)$ Coping with academic stress $(t=2,802$ Sig. $=, 014)$ and reactions to academic stress $(t=-4.614 \mathrm{Sig} .=$, 000). When looking for the effects at reactions to academic stress it was found that levels of cognition, program variable, not only predicts them better (Linear R2 $=0.669$ ) but that acts as a mediating variable of coping of academic stress and reactions to stress academic (BootLLCl $=-.7933$ BootULCl $=-$. and indirect effect of -.4439). Checking the effectiveness of the program and the possibility of develop this theory.
\end{abstract}

Keywords: Training program of skills, soft skills, cognition embodied, interoception.

Historial del artículo:

Recibido, 08 de mayo de 2017; aceptado, 30 de mayo de 2017; disponible en línea, 25 de junio de 2017

* Investigador junior del Centro de Investigación de Servicios Psicológicos de la Universidad Continental.

Correo: giancarlomagrolazo@gmail.com 


\section{INTRODUCCIÓN}

El concepto actual utilizado de habilidades blandas se refiere a un conjunto de habilidades no-cognitivas esenciales para el aprendizaje y el desempeño laboral. (Lagos, 2012). Lo que nos interesará en esta parte introductoria del informe es la supuesta "no-cognición" de estas habilidades. A propósito, una definición más precisa sería la del banco de desarrollo de Latinoamérica que se refiere a Las habilidades cognitivas como la capacidad de una persona de "interpretar, reflexionar, razonar, pensar de manera abstracta y asimilar ideas complejas, resolver problemas y generalizar de lo que se aprende" y a las habilidades no-cognitivas como la capacidad de una persona "de relacionarse con otros y consigo mismo, comprender y manejar las emociones, establecer y lograr objetivos, tomar decisiones autónomas y confrontar situaciones adversas de forma creativa $y$ constructiva. (Ortega, 2016) El informe no ahonda la diferencia entre "lo cognitivo" y lo "no cognitivo" más sí su relación y señala que, en la práctica, los dos tipos de habilidades pueden, y a menudo lo hacen, interactuar y complementarse entre sí. Punto que nos dará paso a la importancia de desarrollar teorías, como la cognición corporizada, que permita el diálogo y la integración más allá del dualismo e ir abandonando la creencia absoluta de que estamos formados por una parte "cognitiva" y una parte "no cognitiva". Sin embargo, antes de dar este paso vamos a conocer un poco más sobre las habilidades blandas.

No cabe duda de que el estudio de habilidades blandas es una tendencia actual, por ejemplo (Deming, 2017) encontró que las ocupaciones con mayor requerimientos de habilidades sociales tendían a emplear a más mujeres el día de hoy en comparación al año 2018, y que el fenómeno reverso es también cierto relativamente con respecto al descenso de empleos en ocupaciones rutinarias. Para agrupar las habilidades blandas se recurre a los modelos de rasgos de personalidad y usualmente el de "los cinco grandes" (Goldberg, 1981) que son Apertura a las nuevas experiencias, Responsabilidad, Extraversión, Afabilidad y Estabilidad emocional. A este modelo algunos investigadores realizan modificaciones; sin embargo, es el mejor punto de partida hasta la fecha. No en vano es el utilizado por el Banco Mundial para la evaluación de habilidades en general empleados y amas de casa en el llamado método STEP. (Pierre, Sanchez Puerta, VAlerio, \& Rajadel, 2014) Estos rasgos se han estudiado descriptiva y longitudinalmente mostrando que, por ejemplo, la extraversión se correlaciona con la inteligencia cristalizada prediciendo el logro académico más que cualquier otra faceta de la inteligencia. (Aimlund, Lee Duckworth, Heckman, \& kautz, 2011) Se encontró también que dos de los cinco grandes, apertura a la experiencia y responsabilidad tenían efectos significativos en la probabilidad de casarse. Mientras que la apertura a la experiencia correlacionaba de forma inversa, la responsabilidad lo hacía de forma directa tanto para varones como para mujeres (Lundberg, 2012)

Más allá de los cinco grandes se han realizado otros estudios donde se ha podido observar la relación de un factor de la personalidad con respecto a la predictibilidad de diversos resultados, por ejemplo, encontraron que el factor diferencial en aquellos personas que aprobaron el examen GED, que es una prueba alternativa equivalente al de estudios en secundario en los Estados Unidos, y que habían abandonado la secundaria y aquellas personas que habían obtenido el grado académico culminando todos los años de estudios secundarios, no era la habilidad cognitiva pues en ambos grupos no se encontró diferencia significativa, sino un factor de personalidad que llevaba, a aquellos que habían abandonado la secundaria, a ser más propensos a fumar y beber, involucrarse en conductas violentas y comportamiento criminal. (Heckman, Eric Humphries, \& Mades, 2010) Lo particular fue que, al hacer un seguimiento a aquellos que aprobaron el examen GED y tenían este factor de personalidad, se encontró que, alrededor del $40 \%$, solo un $3 \%$ a $4 \%$ obtuvieron un diploma de bachillerato a la edad de 40 años. Sin embargo, uno de los mayores estudios realizados a la fecha ha sido el de la Organización para la Cooperación y el Desarrollo Económico analizando las habilidades nocognitivas y su relación con diversas variables como la obesidad, la depresión, problemas de conducta, generar bullyng, ser victimizado, satisfacción con la vida, estilos de vida, entre muchas más, encontrándose relaciones significativas en cada uno de estos pares. (OECD, 2015).

Resalta entonces la importancia de la investigación en habilidades blandas por su estrecha relación a diversas variables pertenecientes al ámbito de la salud en general y, además, porque países como Finlandia o Corea del Sur han complementado las competencias específicas con el desarrollo de habilidades blandas, logrando programas de educación escolar básica que están entre los más reconocidos del mundo y que tienen, además, como fin último la formación de ciudadanos responsables que aportan al progreso de sus naciones. (CEPLAN, 2014).

\section{Habilidades blandas corporizadas}

Ahora bien, se han mencionado estudios descriptivos, sin embargo, aún queda revisar el estado de los métodos y formas para entrenar y cultivar las habilidades blandas. Al respecto podemos encontrar algunos ejemplos en los que, luego de una rigurosa revisión teórica, se han construido matrices de competencias sociales clave como la matriz CASEL que incluye las competencias del darse cuenta de uno 
mismo y los otros, las actitudes positivas y los valores, la toma de decisiones responsable y las habilidades de interacción social. De la misma manera, luego de construir estas matrices se propone un estudio piloto como el caso del programa MASS. (Kechagias, 2011) La presente investigación seguirá el mismo proceso, en un primer momento abordaremos la teoría desde donde se justifica una matriz de desarrollo de competencias y luego describiremos el estudio cuasiexperimental.

Estudios han encontrado que, en el éxito económico de los países y personas, existe una fuerte predicción tanto de los niveles de escolaridad; así como del cambio producido en los años de escolaridad. (Hanushek \& Woessmann, 2008) Es decir, tanto las habilidades cognitivas, como las no cognitivas tendrían una asociación, aún no comprendida del todo, con el éxito futuro. Esto nos lleva de regreso al inicio de este informe y enfatiza el aporte que, precisamente, busca dar este estudio cuasi-experimental. Es decir, comprender la relación entre lo cognitivo y lo no-cognitivo de las habilidades, y para esto vamos a recurrir a la teoría de la cognición corporizada.

La forma clásica de abordar la cognición es la que parte desde los inicios de las ciencias cognitivas y que es válida para comprender los procesos computacionales, a decir el modelo computacional de la mente. El cual está constituido por representaciones mentales y procesos mentales. La forma en la que conocemos, desde este punto de vista, es a través de lógica, reglas, conceptos, analogías e imágenes (Thagard, 2008) y sería absurdo creer que es solo un proceso de alimentación de datos cual si el ser humano fuera un ordenador, pues con el arribo de la neurociencia y la neuroplasticidad se tienen pruebas de que el cerebro se autorregula de manera interconectada a estas señales para generar una predicción, lo que a la larga determina la forma particular de organizarse ante una misma realidad. (Varela, Lachaux, Rodriguez, \& Martinerie, 2001) (Damasio, 1997) esto permite establecer que se conoce a través un diálogo entre la realidad y el organismo, sin embargo aún es un diálogo meramente computacional pues lo que cambia es la complejidad de la respuesta.

La cognición corporizada reposa en la noción de que la cognición se produce en enacción, es decir que, resultado de este diálogo previamente mencionado, emerge un mundo y emerge porque el diálogo no tiene como fin la respuesta, o el reflejo del mundo, sino la acción misma, o la construcción del mundo (Varela, Conocer, 1988). De este modo ya no hablamos de lo cognitivo y de lo no-cognitivo sino de la acción ininterrumpida de los organismos que, a diversos niveles, van adquiriendo consciencia de estar en una realidad emergente, la cual parte desde la realidad de una encarnación o Corporización. Así, se retoma el sentido común en las ciencias cognitivas, pues una habilidad sea cognitiva o no-cognitiva tiene como fin último la construcción de un mundo, lo que rescata el valor de la intencionalidad y la consciencia de la misma.

Esto nos permite construir una teoría de las habilidades que priorice la consciencia como la plataforma del desarrollo sincrónico de lo que conocemos como cognitivo y no-cognitivo. Para abordar el estudio de la conciencia podemos recurrir en un primer momento a la fenomenología, (Butz \& Kutter, 2017) La cual se puede aplicar a la intencionalidad para comprender que un estado intencional se mantiene siempre en referencia a algo pues uno no es simplemente consciente de un objeto, sino es siempre consciente de una forma particular con una cierta perspectiva o punto de vista del objeto. (Gallagher \& Zahavi, 2012) Es decir, que toda habilidad ha de ser comprendida no solo desde sus co-relatos evidentes, sino desde la experiencia consciente de la persona que puede contemplar la relación o cualidad intencional que establece con el objeto. Esto nos establece la posibilidad de un estudio no solo en tercera persona, sino también desde la primera persona. Este enfoque es el llamado enfoque de neurofenomenología. (Montero Anzola, 2008) Como refiere Francisco Varela, la falta de conciencia no es un problema en sí misma, sino lo es la falta de discriminación y la aprehensión de la habitual tendencia al apego (Varela, Thompon, \& Rosch, 1993) la cual puede distorsionar la percepción del mundo que emerge desde las habilidades.

Ahora bien, si queremos validar métodos de investigación en primera persona, hemos de determinar la unidad de estudio, a decir la ya mencionada cualidad intencional o la relación, para luego determinar la vía o herramienta para estudiar dicho objeto. Para esto vamos a retomar la noción de Corporización o encarnación donde tenemos que podemos entender las operaciones cerebrales desde la predicción y corrección del error o codificación predictiva, cuando nos referíamos al diálogo entre la realidad y al organismo en párrafos anteriores, a la interocepción o las condiciones fisiológicas del cuerpo, y así entender un modelo de la emoción como una predicción o inferencia interoceptiva (Seth \& Critchley, 2013).

Esta predicción interoceptiva permite resaltar el contenido emocional como las inferencias de las causas internas y externas que producirían cambios en el cuerpo, lo que sincroniza a estas inferencias con el contexto social (Frith \& Frith, 2012) para explicar el comportamiento desde la emoción, pues al emitir un comportamiento se confirmaría la predicción en la respuesta del contexto, lo que podría mantener este lenguaje interoceptivo aún este sea un comportamiento inadaptado en base a un error predictivo, como 
Tabla 1

Correlación, estado de ánimo y rendimiento académico.

\begin{tabular}{|c|c|c|c|c|c|c|}
\hline Factor & $\begin{array}{l}\text { Habilidades } \\
\text { cognitivas }\end{array}$ & $\begin{array}{c}\text { Acción } \\
\text { (ejemplos) }\end{array}$ & Relación & $\begin{array}{l}\text { Habilidades } \\
\text { no cognitivas }\end{array}$ & $\begin{array}{l}\text { Inferencia } \\
\text { Interoceptiva }\end{array}$ & $\begin{array}{l}\text { Método de } \\
\text { entrenamiento }\end{array}$ \\
\hline Partida & $\begin{array}{l}\text { Sensorio- } \\
\text { motricidad } \\
\text { Psicológicas } \\
\text { Socio- } \\
\text { contextuales }\end{array}$ & $\begin{array}{l}\text { Tocar un } \\
\text { objeto } \\
\text { Recordar } \\
\text { Comparar } \\
\text { Reconocer }\end{array}$ & $\begin{array}{l}\text { (Ver el objeto) } \\
\text { Aceptación } \\
\text { incondicional } \\
\text { / Aceptación } \\
\text { condicionada }\end{array}$ & $\begin{array}{l}\text { Honestidad } \\
\text { Optimismo } \\
\text { Creatividad } \\
\text { Apertura a la } \\
\text { experiencia }\end{array}$ & $\begin{array}{l}\text { Reconocer } \\
\text { el dolor } \\
\text { emocional }\end{array}$ & $\begin{array}{l}\text { Meditación } \\
\text { Shamata } \\
\text { Dinámicas de } \\
\text { movilización y }\end{array}$ \\
\hline Desarrollo & Científicas & $\begin{array}{l}\text { Hipotetizar } \\
\text { Comprobar } \\
\text { Replantear } \\
\text { elementos } \\
\text { y procesos }\end{array}$ & $\begin{array}{l}\text { (Relación } \\
\text { a través de } \\
\text { la acción) } \\
\text { Comprensión } \\
\text { /control }\end{array}$ & $\begin{array}{l}\text { Estabilidad } \\
\text { Emocional } \\
\text { Sociabilidad } \\
\text { Empatía } \\
\text { Adaptación } \\
\text { al cambio } \\
\text { Paciencia }\end{array}$ & $\begin{array}{l}\text { Integrar el } \\
\text { dolor } \\
\text { emocional }\end{array}$ & $\begin{array}{l}\text { exploración } \\
\text { sensorial } \\
\text { Meditación } \\
\text { Vipassana } \\
\text { Juego e } \\
\text { improvisación } \\
\text { para }\end{array}$ \\
\hline Mantención & $\begin{array}{l}\text { Morales } \\
\text { Trans- } \\
\text { personales }\end{array}$ & $\begin{array}{l}\text { Defender la } \\
\text { verdad } \\
\text { Resistir a la } \\
\text { violencia } \\
\text { Perdonar la } \\
\text { vida }\end{array}$ & $\begin{array}{l}\text { (Interrelación } \\
\text { entre los } \\
\text { objetos) } \\
\text { Sabiduría / } \\
\text { Ignorancia }\end{array}$ & $\begin{array}{l}\text { Responsabilidad } \\
\text { Ética } \\
\text { Defensa y } \\
\text { compromiso } \\
\text { (amor) }\end{array}$ & $\begin{array}{l}\text { Trascender } \\
\text { el dolor } \\
\text { emocional }\end{array}$ & $\begin{array}{l}\text { enfrentar el } \\
\text { temor a fallar } \\
\text { Meditación } \\
\text { Prajna } \\
\text { Práctica en } \\
\text { los contextos } \\
\text { particulares }\end{array}$ \\
\hline
\end{tabular}

en el caso del apego. Este error en la integración interoceptiva y exteroceptiva se ha explorado con el experimento de la mano de goma el cual ha tenido variaciones usando realidad virtual en la que se ha sincronizado los latidos del corazón del individuo con el falso brazo que se puede apreciar desde los dispositivos virtuales (Suzuki, Garfinkel, Critchley, \& Seth, 2013). También podríamos ver este error en las disfunciones relacionadas a la ansiedad. (Paulus \& Stein, 2006).

Así tenemos que, para la construcción de una teoría de las habilidades que priorice la consciencia como la plataforma del desarrollo sincrónico de lo que conocemos como cognitivo y no-cognitivo, establecemos un método de comprobación: Correlatos de observaciones en tercera y en primera persona (neurofenomenología), luego una unidad de estudio: La relación o cualidad intencional y, finalmente, una herramienta de investigación que sería la conciencia interoceptiva. Esta teoría está resumida en la Tabla 1, la cual además provee la matriz para la construcción del programa.

En un primer momento, en un factor llamado "partida", esta teoría enfatiza la percepción interoceptiva desde un entrenamiento que en la tradición budista se describe como calma o Shamata (Harvey, 2015) y entrenamiento en dinámicas de movilización y exploración sensorial. para desarrollar el arte de diferenciar lo que hay y lo que no en el momento presente, esta sería la cualidad intencional que determinaría que una habilidad se siga cultivando o no, lo que impulsa sincrónicamente el desarrollo cognitivo y no cognitivo a determinado nivel.

En un segundo momento, llamado "desarrollo" la teoría enfatiza la percepción interoceptiva desde un entrenamiento que en la tradición budista se describe como Vipassana o ver las cosas como son, lo cual está básicamente referido al dolor o sufrimiento, por lo que se entrena también a través del juego o la improvisación que da licencia para fallar y equivocarse. La cualidad intencional que impulsa el desarrollo de lo no cognitivo y lo cognitivo sería, precisamente, ver más allá del sufrimiento (Nhat Hanh, 2000), lo que llevaría a desarrollar las habilidades más allá de las complicaciones que se presenten en el contexto.

Finalmente en un tercer y último momento, llamado "mantención", la teoría enfatiza la percepción interoceptiva con la maestría del entrenamiento de Samatha y Vipassana, lo que acerca al prajna - la capacidad de ver en el meollo de la materia, no en calidad de resultado de la erudición, sino como consecuencia de haber renunciado a todo lo que oscurece una percepción clara. Es decir, que el verdadero problema no es el sufrimiento, sino la ignorancia. Esta conciencia sería el punto definitivo donde lo cognitivo, las representaciones del mundo, y lo no cognitivo, el mundo, se encuentran. (Brazier, 2003)

Se busca el desarrollo de estos tres factores, Reconociendo la inter-relación entre los objetos o lo que se conoce en ciencias cognitivas como el fenómeno local-global de la emergencia de la realidad (Cullen, 2011) y que tiene un amplio ámbito de exploración en lo que se conoce actualmente como ecología cognitiva (Hutchins, 2010). 


\section{MATERIAL Y MÉTODOS}

\section{Programa de intervención}

Como se mencionó en el párrafo anterior, el objetivo de establecer una teoría era construir una matriz que organice el entrenamiento y la capacitación de habilidades blandas, esta matriz permitió la construcción del programa piloto llamado "La paz en cada paso" pues hace referencia al poder encontrar los recursos necesarios en cada paso, el cual relaciona al cuerpo del caminante con el camino que se recorre, lo que es en sí la esencia del modelo cognitivo de enacción (cognición corporizada). El programa constó de tres workshops de mindfulness, así como seis sesiones complementarias a través de dinámicas Gestalt y juegos teatrales para la exploración del movimiento y la expresión.

\section{Muestra}

El diseño fue de tipo cuasi-experimental con grupo control no equivalente; el muestreo fue de tipo no probabilístico accidental pues los participantes decidieron, luego de una convocatoria por diversos salones de la universidad, participar en la entrevista inicial del programa, cuyo criterio de inclusión fue estar cursando estudios universitarios y haber experimentado cierto nivel de nerviosismo durante el actual o último periodo de estudios. El criterio de exclusión fue percibirse con una mala salud al momento de iniciar el programa.

El total de participantes del programa fue de 22 personas, de estas 13 conformaron el grupo experimental $y$, aquellos que, por razones de tiempo y prioridades no pudieron tomar las evaluaciones iniciales ni llevar al programa accedieron a ser el grupo control (9 personas) y pudieron tomar las evaluaciones semanas después lo que coincidió con el fin del trabajo experimental, con el compromiso por parte del investigador de ser incluidos en versiones posteriores del programa.

\section{Medición}

Para medir la efectividad del programa se hizo uso de los siguientes instrumentos:

Inventario SISCO del estrés académico, de Arturo Barraza Macias, (Barraza, 2008) cuyo objetivo es la medición de tres componentes sistémico-procesuales del estrés académico: estímulos estresores (input), síntomas (indicadores del desequilibrio sistémico) y estrategias de afrontamiento (output). El cual, en su proceso de elaboración obtuvo obtuvo una confiabilidad por mitades de .87 y una confiabilidad en alfa de Cronbach de. 90
Escala de afrontamiento del estrés académico (a-cea) (Cabanach, Valle, Rodriguez, Piñeiro, \& Freire, 2010) cuyo objetivo es identificar las estrategias de afrontamiento del estrés utilizadas por los estudiantes en el ámbito universitario (Reevaluación positiva, Búsqueda de apoyo, Planificación). El cual, en su proceso de elaboración obtuvo .864 (Alpha de Cronbach) en la versión original.

Mindfull Attention Awareness Scale (MAAS) de los autores Kirk Warrern y Richard Ryan de la Universidad de Virgina Commonwealth, cuyo objetivo es el medir el rasgo de la atención plena (La disposición atencional hacia lo que ocurre en el momento presente), característica única de la conciencia que está relacionada con y es predictiva de varios constructos de la autorregulación y el bienestar. El cual en su proceso de elaboración obtuvo un 0.89 de Alfa de Cronbach en la adaptación española.

Inventario de Niveles de Cognición FV del autor de esta investigación (Magro, 2017) cuyo objetivo es evaluar Niveles de cognición o lo que le es dado conocer a un ser humano desde una realidad corporizada. Siendo estos, los niveles egoístas, socio-céntrico, existencial y compasivo. El inventario se encuentra en su versión exploratoria donde alcanza 0,88 de Alpha de Cronbach. Este inventario se relaciona con la variable que entrena el programa de habilidades blandas.

Estos datos fueron procesados con IBM Statistics y la aplicación Process 16.3

\section{RESULTADOS}

Se llevaron a cabo mediciones con los instrumentos mencionados a todos los participantes del programa antes de asignarlos a los grupos control y experimental, y por, lo tanto, antes de la intervención mediante el programa. Obteniendo los siguientes resultados descriptivos:

Los participantes del grupo experimental en el pretest obtuvieron con respecto a niveles de nerviosismo $(M=3,2 \mathrm{Ds}=, 78)$, Atención plena $(M=61,1 \mathrm{Ds}=$ 18,88), Cognición $(M=66,4 \quad D s=13,11)$, Total afrontamiento $(M=74,4 \mathrm{Ds}=13,15)$ Total Reacciones $(M=40,9 \mathrm{Ds}=8,78)$

Los participantes del grupo experimental en el posttest obtuvieron con respecto a niveles de nerviosismo $(M=2,23 \quad D s=1,23)$, Atención plena $(M=75,3$ $\mathrm{Ds}=7,53)$, Cognición $(M=77,84 \mathrm{Ds}=12,53)$, Total afrontamiento $(M=84,50 \mathrm{Ds}=15,04)$ Total Reacciones $(M=34,30 \mathrm{Ds}=9,73)$

Los participantes del grupo control en el post-test obtuvieron con respecto a niveles de nerviosismo 
$(M=3,8 \quad D s=1,05)$, Atención plena $(M=50,7 \quad D s=$ 16,36), Cognición $(M=45,88 \quad D s=13,58)$, Total afrontamiento $(M=62,88 \mathrm{Ds}=13,15)$ Total Reacciones $(M=56,22 \mathrm{Ds}=11,72)$

Al observar los efectos del programa con respecto a las variables que se pueden medir con el test SISCO del estrés académico, tenemos que, con respecto a los niveles de nerviosismo, como se observa en la figura 1 , se observa una diferencia en el pre-test y post-test del grupo experimental de 1 punto en promedio, si consideramos que la escala es de 5 puntos, la diferencia es considerable, lo que se comprueba con la prueba $t$ de muestras emparejadas (Sig. $=009, \mathrm{t}=$ $3,122)$; y con respecto a la medición post test del grupo experimental con el post-test del grupo control se encuentra una diferencia de 1, 55 puntos en promedio, cuya significancia estadística se comprueba con la prueba $t$ de muestras independientes $(\mathrm{Sig} .=003$, $t=-3,379)$.

Con respecto a la variable reacciones al estrés, tal como se puede observar en la figura 2, existe una

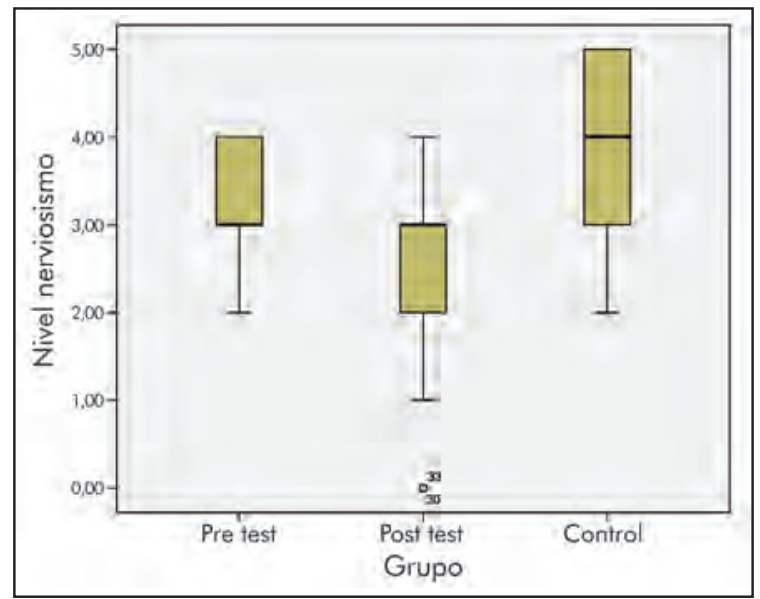

Figura 1. Comparación Medias (Nerviosismo).

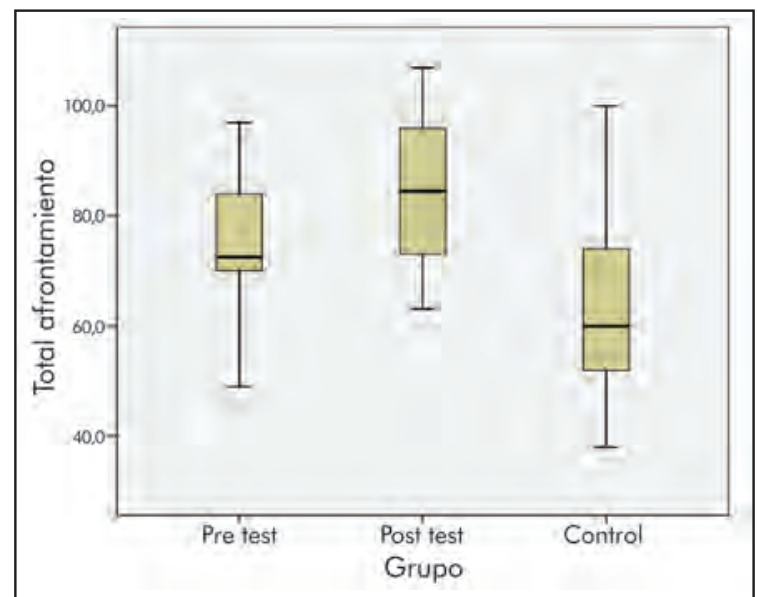

Figura 3. Comparación Medias (Afrontamiento al estrés). diferencia de 10 puntos de reducción en promedio de una escala de aproximadamente 80 puntos con respecto a las mediciones pre-test y post-test en el grupo experimental. Las diferencias significativas se repiten en el indicador total y los sub-indicadores al realizar la prueba $\dagger$ de muestras emparejadas: Total reacciones (Sig. $=0,008 ; \dagger=-3,144$ ) Reacciones físicas. (Sig. $=0,42 ; \dagger=-2,275$ ) Reacciones psicológicas (Sig. $=0,002 ; t=-3,926)$ Reacciones comportamentales (Sig. $=0,34 ; \dagger=-2,385$ ) Y con respecto a la medición post test del grupo experimental con el post-test del grupo control se encuentra una diferencia de 22 puntos en promedio cuya significancia estadística se comprueba con la prueba t de muestras independientes, en el indicador total y los sub-indicadores, Total reacciones (Sig. $=0,000 ; \dagger=-4,614$ ) Reacciones físicas. (Sig. $=0,001 ; t=-4,085) \quad$ Reacciones psicológicas (Sig. $=0,000 ; \dagger=-5,494$ ) Reacciones comportamentales (Sig. $=0,003 ; t=-3,522$ ).

Con respecto a la variable "afrontamiento al estrés académico" que se pudo medir con el test A-CEA, tal como se puede observar en la Figura 3 existe una

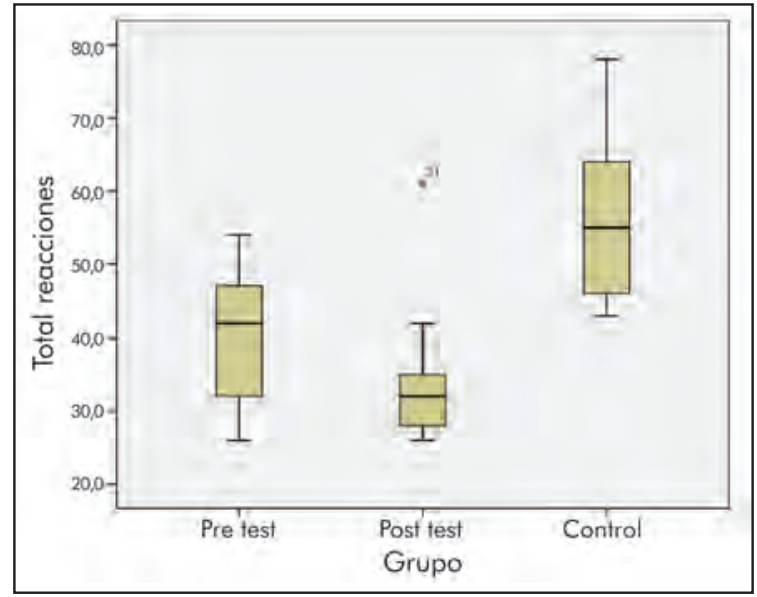

Figura 2. Comparación Medias (Reacciones al estrés).

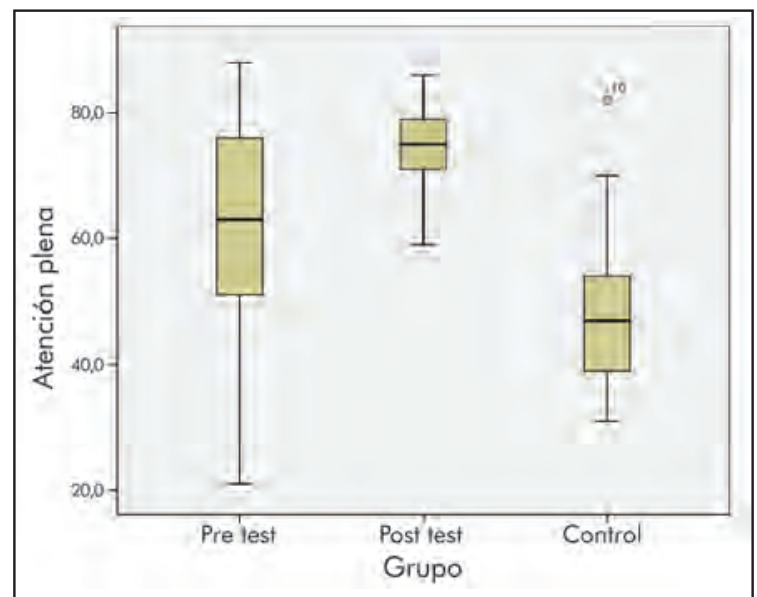

Figura 4. Comparación Medias (Atención plena). 


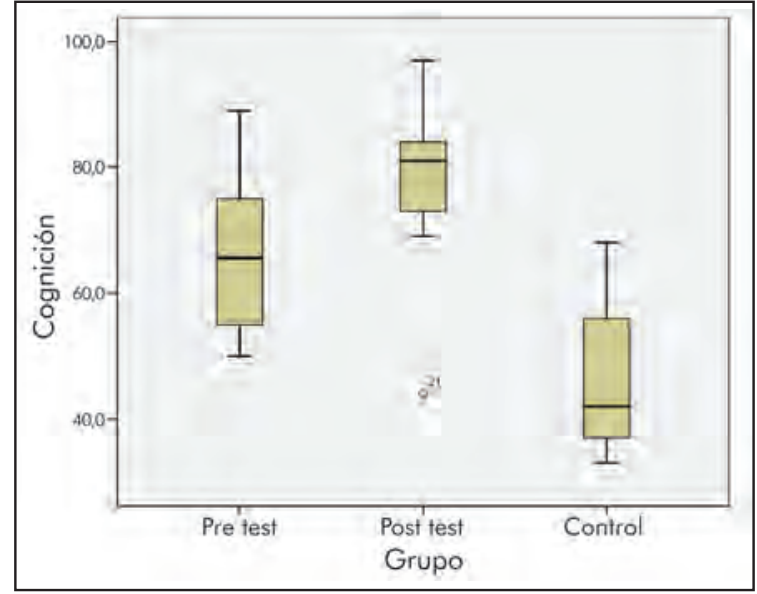

Figura 5. Comparación Medias (Cognición).

diferencia de aproximadamente 12 puntos en promedio en una escala de 90 puntos aproximadamente, con respecto a las mediciones pre-test y post-test en el grupo experimental. Las diferencias significativas se exploraron en el indicador total y los sub-indicadores al realizar la prueba $t$ de muestras emparejadas: Total afrontamiento (Sig. $=0,034 ; t=2,425$ ) Búsqueda de apoyo (Sig. $=0,32 ; t=-2,462$ ) Reevaluación positiva (Sig. =0,133; $t=1,622$ ) Planificación (Sig. =0, 132; $t=1,462) \quad Y$ con respecto a la medición post test del grupo experimental con el post-test del grupo control se encuentra una diferencia de 22 puntos en promedio cuya significancia estadística se comprueba con la prueba + de muestras independientes, en el indicador total y los sub-indicadores, Total afrontamiento (Sig. =0,14; $t=2,802)$ Búsqueda de apoyo (Sig. =0,54; $t=-2,144) \quad$ Reevaluación positiva (Sig. $=0,016$; $\dagger=2,661$ ) Planificación (Sig. $=0,021 ; \dagger=2,535$ ).

Con respecto a la variable atención plena, esta se pudo medir con el test MAAS de atención plena y, tal como se puede observar en la Figura 4 existe una diferencia de 13 puntos en promedio en una escala.

De 80 aproximadamente con respecto a las mediciones pre-test y post-test en el grupo experimental, la diferencia significativa se exploró en el indicador total mediante la prueba $\dagger$ de muestra emparejada (Sig. $=0,022 ; t=2,628)$ y con respecto a la medición post test del grupo experimental con el post-test del grupo control se encuentra una diferencia de 25 puntos en promedio cuya significancia estadística se comprueba con la prueba t de muestras independientes (Sig. $=0,000 ; \dagger=4,762$ ) Figura 4: Comparación Medias (Atención plena)

Finalmente, respecto de la variable cognición tal como se puede observar en la figura 5 existe una diferencia de aproximadamente 34 puntos en promedio en una escala de 100 puntos con respecto a las mediciones pretest y post-test en el grupo experimental. Las diferencias significativas se exploraron en el indicador total y los sub-indicadores al realizar la prueba $\dagger$ de muestras emparejadas: Cognición total (Sig. $=0,012 ; t=2,950$ ); Información (Sig. =0,039; $\quad t=2,317$ Transformación (Sig. $=0,014 ; t=2,856)$ Individualidad (Sig. =0,01 1; $t=2,992$ ) Intersubjetividad (Sig. $=0,046 ; t=2,223$ ). Con respecto a la medición post test del grupo experimental con el post-test del grupo control se encuentra una diferencia de 34 puntos en promedio cuya significancia estadística se comprueba con la prueba $\dagger$ de muestras independientes, en el indicador total y los subindicadores. Cognición total (Sig. $=0,000 ; t=5,560$ ) Información (Sig. $=0,000 ; t=5,018$ ) Transformación (Sig. $=0,000 ; t=5,187$ ) Individualidad (Sig. $=0,000$; $t=6,230$ ) intersubjetividad (Sig. $=0,159 ; t=1,521$ ).

\section{Predictores de las reacciones al estrés}

Para este fin se llevó el análisis de regresión lineal Simple de diversas variables, cada una por separado, con las reacciones al estrés académico para comprobar cuál de ellas funciona como un mejor predictor por si sola. Los resultados fueron los siguientes: Fuentes de estrés académico $\left(F=, 002 R^{2}=0,300\right)$; Atención plena $\left(F=0,001 R^{2}=0,329\right)$; Afrontamiento $(F=0,005$ $\left.R^{2}=0,241\right)$ y Cognición $\left(F=0,000 R^{2}=0,671\right)$. Con respecto a esta última variable se muestra además la Figura 6 donde se muestra el coeficiente $\beta,-569$ y la significación T-test 0,000 para establecer el rol de la Cognición como predictor.

Se llevaron a cabo correlaciones bivariadas de los subindicadores tanto del afrontamiento al estrés como de los niveles de cognición, así como regresiones múltiples de estos factores con las reacciones al estrés.

Con respecto a los sub-indicadores del afrontamiento al estrés académico. Los coeficientes de correlación Pearson fueron: Reevaluación positiva $(-, 403)$

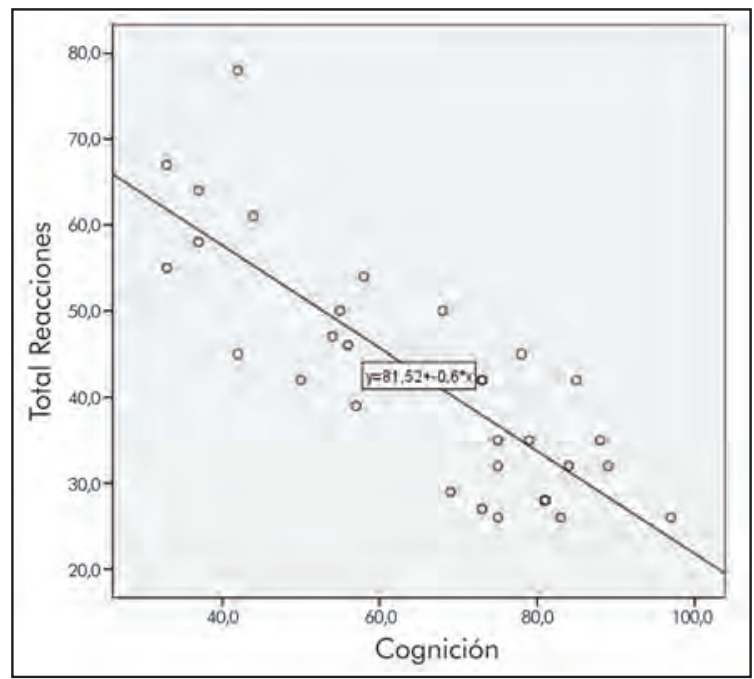

Figura 6. Regresión cognición.- Reacciones al estrés. 
Tabla 2

Regresión múltiple Cognición.- Reacciones al estrés.

\begin{tabular}{|c|c|c|c|c|c|c|}
\hline \multicolumn{7}{|c|}{ Coeficientes $^{a}$} \\
\hline \multirow[b]{2}{*}{ Modela } & & \multicolumn{2}{|c|}{$\begin{array}{l}\text { Coeficientesnoo } \\
\text { estandarizados }\end{array}$} & \multirow{2}{*}{$\begin{array}{c}\text { Coeficientes } \\
\text { estandarizado } \\
\text { s } \\
\text { Beta } \\
\end{array}$} & \multirow[b]{2}{*}{$t$} & \multirow[b]{2}{*}{ Sig. } \\
\hline & & B & Error estándar & & & \\
\hline \multirow[t]{5}{*}{1} & (Constante) & 80,062 & 6,253 & & 12,805 & .000 \\
\hline & INFORMACIÓN & -.578 & 344 &,- 351 & $-1,682$ & 104 \\
\hline & TRANSFORMACIÓN &,- 728 &, 293 &,- 375 & $-2,484$ & .019 \\
\hline & INDIVIDUAL &,- 221 &, 723 &,- 061 &,- 305 &, 762 \\
\hline & $\begin{array}{l}\text { INTERSUBJETIVIDA } \\
\text { D }\end{array}$ & -609 & 442 & -180 & $-1,377$ & .180 \\
\hline
\end{tabular}

Búsqueda de apoyo $(-, 285)$ Planificación $(-, 557)$. Al realizar el análisis de correlación múltiple se determinó que juntos determinan en ,316 R2 las reacciones físicas al estrés con un 2,065 en la prueba DurbinWatson y de los tres indicadores es la planificación la que mejor predice inversamente las reacciones al estrés. $\beta,-1,210$ y la significación T-test ,030.

Con respecto a los sub-indicadores de los niveles de cognición. Los coeficientes de correlación Pearson fueron: Información (-,747), Transformación $(-, 728)$ individualidad $(-, 677)$ e Intersubjetividad $(-, 560))$. Al realizar el análisis de correlación múltiple se determinó que juntos determinan en , 677 R2 las reacciones físicas al estrés con un 2,644 en la prueba Durbin-Watson y de los cuatro indicadores, tal como se observa en la Tabla 2, es la transformación la que mejor predice inversamente las reacciones al estrés. $\beta,-, 728$ y la significación T-test, 019 .

Finalmente, se llevó a cabo el análisis de mediación para comprobar el efecto que tiene los niveles de cognición como mediadora entre el afrontamiento al estrés académico y las reacciones al estrés. Cómo se observa en la figura 7 , el efecto que tiene el afrontamiento al estrés en los niveles de cognición es significativo $(B=0.69, t=4, .87, p=.00)$ de la misma manera, el efecto que tiene los niveles de cognición en las reacciones al estrés es significativo $(B=-0.64$, $t=-5.96, p=.00)$. El efecto total del afrontamiento al estrés en las reacciones al estrés es significativo $(B=-0.369, \dagger=-3,03, p \ldots .005)$ y se comprueba estadísticamente la mediación de los niveles de cognición entre el afrontamiento al estrés académico y las reacciones al estrés académico (BootLLCl=-.7933 BootULCI $=-.2247$ y efecto indirecto de -.4439 ).

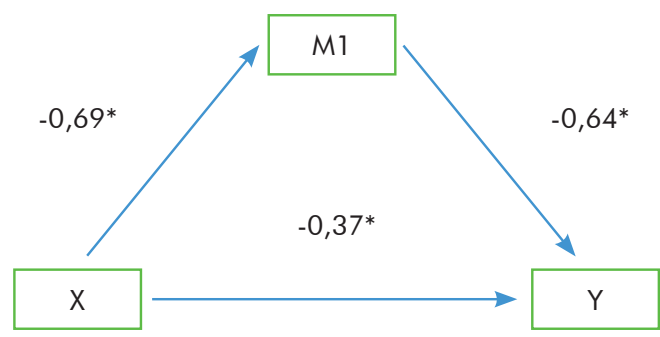

$X=$ Afrontamiento

M1 = Niveles de cognición

$Y=$ Reacciones al estrés * $\mathrm{p}<0,05$

\section{DISCUSIÓN}

El programa (habilidades blandas corporizadas) ha tenido un efecto estadísticamente significativo con respecto a la reducción de los niveles de nerviosismo en los participantes, reacciones (síntomas) de los participantes al estrés académico, siendo las reacciones psicológicas las de mayor diferencia y finalmente, se ha observado un incremento estadísticamente significativo en la atención plena de los participantes. Esto es coherente a las investigaciones citadas que evidencian la relación de las habilidades blandas con variables de bienestar (Deming, 2017) (Hanushek \& Woessmann, 2008) (OECD, 2015). El incremento de las habilidades blandas en este programa se comprueba no solo a nivel del incremento estadísticamente significativo de habilidades de afrontamiento al estrés académico, lo cual incluye capacidades de búsqueda de apoyo, reevaluación positiva y planificación; sino también un incremento estadísticamente significativo en los niveles de cognición, variables de entrenamiento del programa que son coherentes a la matriz mostrada en este artículo y que se comportan en los procesos de regresión como mejor predictor de variables de bienestar. Esta variable incluye, en el eje información, habilidades que buscan ampliar la percepción y aceptación de objetos; eje transformación, referido a 
habilidades para la toma de acción como vehículo de desarrollo; eje individualidad, referido a habilidades para identificar y defender la propia identidad; y eje intersubjetividad, referido a habilidades para establecer relaciones que impulsen el desarrollo de todos los elementos. Siendo un paso significativo en el desarrollo de la teoría de la cognición corporizada, expuesta en este artículo.

Finalmente, para clarificar el rol predictivo tanto del afrontamiento como de los niveles de cognición, se ha realizado un análisis de mediación donde se evidencia estadísticamente que la cognición tiene un rol de mediación entre el afrontamiento al estrés académico y las reacciones al estrés académico, es decir que el afrontamiento eficaz al estrés tendría un efecto directo en la reducción de síntomas o respuestas (físicas, psicológicas y comportamentales) al estrés como se ha demostrado en investigaciones (Zarej, Hasjemi, Sadipoor, Delavar, \& Khoshnevisan, 2016) , sino también un efecto indirecto a través de la ampliación de la cognición corporizada en la conciencia del individuo. Es decir conciencia de sus habilidades cognitivas y no cognitivas a través de la interocepción mediante el reconocimiento, integración y trascendencia del dolor emocional.

En conclusión, esta investigación defiende la construcción de una teoría de las habilidades que priorice la consciencia como la plataforma del desarrollo sincrónico de lo que conocemos como cognitivo y no-cognitivo. Los resultados estadísticamente significativos del programa nos permiten sugerir que las habilidades, como las que se observan en el afrontamiento al estrés, no tendrían un efecto en sí mismas; sino porque permiten la ampliación de la conciencia del individuo a nivel corporizado, el cual sería un nuevo punto de discusión con respecto a las habilidades blandas.

\section{REFERENCIAS BIBLIOGRÁFICAS}

Aimlund, M., Lee Duckworth, A., Heckman, J., \& kautz, T. (2011). Personality Psychology and Economics. In E. Hanushek, S. Machin, \& L. Woessman, Handbook of the Economics of Education (pp. 1-181). Amsterdam: Elsevier.

Barraza, A. (2008). El estrés académico en alumnos de maestría y sus variables moduladoras: un diseño de diferencia de grupos. Avances en Psicología Latinoamericana, 270-289.

Brazier, D. (2003). Terapia Zen. Madrid : Oberon.

Butz, M., \& Kutter, E. (2017). how the mind comes into being. Oxford: Oxford University Press.

Cabanach, R., Valle, A., Rodriguez, S., Piñeiro, I., \& Freire, C. (2010). Esala de afrontamiento de estrés académico. Revista iberoamericana de psicología y salud, 51-64.

CEPLAN. (2014). La educación del futuro y el futuro de la educación. Lima: Punto y grafía.

Cullen, M. (2011). Mindfulness-Based Interventions: An Emerging phenomenon. Mindfulness Springer.

Damasio, A. (1997). El error de descartes. Santiago de chile: Andres Bello.

Deming, D. (2017). The growing importance of social skills in labor market. Working Paper 21473.

Frith, C., \& Frith, U. (2012). Mechanisms of Social Cognition. Annual review of psychology, 287-313.

Gallagher, S., \& Zahavi, D. (2012). The phenomenological mind. New York: Routledge.

Goldberg, L. (1981). the search for universals in personality lexicons. Review of personality and social psychology, 141-165.

Hanushek, E., \& Woessmann, L. (2008). The role of cognitive skills in economic development. Journal of economic Literature, 607-668.

Harvey, P. (2015). Introducing Samatha Meditation. Retrieved from Samatha.org: https://www.samatha. org/sites/default/files/Introducing_Samatha_ Meditation ver 2.1 Sept 2015Introduction.pdf

Heckman, J., Éric Humphries, J., \& Mades, N. (2010). the GED. In E. Hanushek, S. Machin, \& L. Woessman, Handbook of the economics of education, Volume 3 (p. 616). Amsterdam: standford.

Hutchins, E. (2010). Cognitive Ecology. Topics in Cognitive Science 2, 705-725.

Kechagias, K. (2011). Teachink and assessing soft skills. Thesalonika: Second Chance School of thessaloniki.

Lagos, C. (2012). Aprendizaje experencial en el desarrollo de habilidades blandas. Santiago de Chile: Universidad Alberto Hurtado.

Lundberg, S. (2012). Personality and marital surplus. IZA Journal of labor economics, 1-21.

Magro, G. (2017). Inventario FV de niveles de Cognición. Huancayo.

Montero Anzola, J. (2008). La Neuro-Fenomenología: Una relfexión sobre las metodologías en primera persona en el estudio de la conciencia. Universitas Philosophica, 115-140.

Nhat Hanh, T. (2000). La paz está en cada paso. santiago de Chile: Bantam books.

OECD. (2015). Skills for Social Progress: The Power of Social and Emotional Skills. OECD.

Ortega, T. (2016). desenredando la conversación sobre habilidades blandas. Washington: Banco de desarrollo de amética latina.

Paulus, M., \& Stein, M. (2006). An Insular View of Anxiety. Biology psychiatry, 383-387.

Pierre, G., Sanchez Puerta, M. L., VAlerio, A., \& Rajadel, T. (2014). STEP Skills Measuremente Surveys. California: world Bank Group.

Seth, A., \& Critchley, H. (2013). Extending predictive processing to the body: Emotion as interoceptive inference. Behavioral and Brain Sciences, 227 - 
228.

Suzuki, K., Garfinkel, S., Critchley, H., \& Seth, A. (2013). Multisensory integration across exteroceptive and interoceptive domains modulates self-experience in the rubber-hand illusion. Neuropsychologia, 29092917.

Thagard P,. (2008). Introducción a las ciencias cognitivas. Madrid: Katze editores.

Varela, F. (1988). Conocer. Barcelona: Gedisa.

Varela, F., Lachaux, J.-P., Rodriguez, E., \& Martinerie, J. (2001). the brain web: Phase synchronization and large scale integration. Nature, 229.239.
Varela, F., Thompon, E., \& Rosch, E. (1993). The embodied Mind. Massachusetts: MIT Press.

Zarej, P., Hasjemi, T., Sadipoor, S., Delavar, A., \& Khoshnevisan, Z. (2016). Effectiveness of Coping Strategies in Reducing Student's Academic Stress. International Journal of Mental Health \& Addiction, 14, 1057-1061. 